\section{IC-P-024 CAPITALIZING ON COMPLEMENTARY FDG-PET AND FLORBETAPIR-PET DATA SETS TO DISTINGUISH BETWEEN EARLY AND LATE MILD COGNITIVE IMPAIRMENT USING MULTIMODAL PARTIAL LEAST SQUARES}

Hillary Protas ${ }^{1}$, Kewei Chen ${ }^{1}$, Cole Reschke ${ }^{1}$, Auttawut Roontiva ${ }^{1}$, Wendy Lee ${ }^{1}$, Mark Nishimura ${ }^{1}$, Napatkamon Ayutyanont ${ }^{1}$, Xiaofen Liu ${ }^{1}$, Pradeep Thiyyagura ${ }^{1}$, Stephanie Parks ${ }^{1}$, Robert Koeppe ${ }^{2}$, William Jagust ${ }^{3}$, Norman Foster ${ }^{4}$, Michael Weiner ${ }^{5}$, Adam Fleisher ${ }^{1}$, Eric Reiman ${ }^{1},{ }^{1}$ Banner Alzheimer's Institute, Phoenix, Arizona, United States; ${ }^{2}$ University of Michigan, Ann Arbor, Michigan, United States; ${ }^{3}$ University of California, Berkeley, Berkeley, California, United States; ${ }^{4}$ University of Utah, Salt Lake City, Utah, United States; ${ }^{5}$ University of California, San Francisco, San Francisco, California, United States.

Background: We previously introduced a voxel-based image analysis algorithm known as multi-modal partial least squares (MMPLS) to characterize the linkage between covarying patterns in two or more complementary complex data sets. Here we used the MMPLS to characterize and compare covarying patterns in FDG PET and florbetapir PET images from 150 patients with early mild cognitive impairment (eMCI) and 78 patients with late MCI (IMCI) in the Alzheimer's Disease Neuroimaging Initiative (ADNI). Methods: MMPLS was used in conjunction with SPM8 to characterize and compare MMPLS subject scores irrespective of the subject's clinical status and free from the Type I error inflation associated with multiple regional comparisons. Brain maps of the between-group differences in covarying FDG PET and florbetapir PET latent variables were compared to maps of between-group differences in FDG PET and florbetapir PET measurements using convention univariate statistics. Results: Differences between the eMCI and IMCI MMPLS subject scores were highly significant (Hotelling 2 sample T 2: $P=2.2 \mathrm{e}-06$, with no need to correct for multiple regional comparisons). By comparison, voxels associated with maximal between group differences in the florbetapir PET and FDG PET brain maps generated using conventional statistics were associated with p-values of $P=3.1 \mathrm{e}-06$ and $9.0 \mathrm{e}-06$, respectively, prior to any correction for multiple regional comparisons and p-values of $P=0.015$ and $P=0.082$ after correction. Between-group florbetapir PET and FDG PET brain maps simultaneously generated using the MMPLS were similar to those generated in independent comparisons with conventional univariate statistics. Conclusions: When applied to FDG and florbetapir images from the same persons, the MMPLS appears to distinguish between eMCI and lMCI patients with improved statistical power and freedom from multiple regional comparisons. Additional studies are needed to clarify the value of voxel-based image analysis algorithms that capitalize on two or more complementary data sets from the same persons in the early detection, tracking, and differential diagnosis of $\mathrm{AD}$ and the evaluation of $\mathrm{AD}$-modifying treatments.

\section{IC-P-025 IN VIVO DETECTION OF AMYLOID DEPOSITION USING [11C]AZD2184 PET}

Hitoshi Shimada ${ }^{1}$, Hitoshi Shinotoh ${ }^{2}$, Shigeki Hirano ${ }^{1}$, Takaaki Mori ${ }^{3}$, Yoko Eguchi $^{4}$, Noriko Tanaka ${ }^{4}$, Fumitoshi Kodaka ${ }^{1}$, Hironobu Fujiwara ${ }^{1}$, Yasuyuki Kimura ${ }^{1}$, Satoshi Kuwabara ${ }^{5}$, Harumasa Takano ${ }^{1}$, Makoto Higuchi ${ }^{4}$, Hiroshi Ito ${ }^{4}$, Tetsuya Suhara ${ }^{4},{ }^{1}$ National Institute of Radiological Sciences, Chiba-shi, Japan; ${ }^{2}$ National Institute of Radiological Sciences, Chiba, Japan; ${ }^{3}$ Ehime University School of Medicine, Toon-city, Ehime, Japan; ${ }^{4}$ National Institute of Radiological Sciences, Chiba, Japan; ${ }^{5}$ Chiba University, Chiba, Japan.

Background: [11 C]AZD2184 has been recently developed as an amyloid imaging PET ligand that has high affinity for beta-amyloid $(\mathrm{A} \beta)$ and low nonspecific binding in the brain. We investigated the characteristics of [11 C]AZD2184 retention in cognitive healthy elderlies and patients with cognitive impairments. Methods: Participants were ten patients with Alzheimer's disease (AD), one patient with frontotemporal dementia (FTD), five patient with mild cognitive impairments (MCI) and age-matched nine healthy controls (HC). A dose (about $10 \mathrm{mCi}$ ) of [11 C]AZD2184 was intra- venously injected and sequential PET scans were performed for $90 \mathrm{~min}$. Standardized uptake value ratio (SUVR) was calculated using cerebellar cortex as the reference region, and SUVR images were visually assessed in each subject. Voxel-wise distribution volume ratio (DVR) was also calculated using Logan plot analysis with the cerebellum as a reference region. Voxel-based group comparison was performed followed by VOI analysis. Results: Visual assessment of SUVR images and quantitative analysis of DVR showed that $\mathrm{A} \beta$ deposition was high in parietal, lateral temporal and frontal cortices, especially in precuneus in the $\mathrm{AD}$ and $\mathrm{MCI}$ groups, and two of nine HC subjects. Seven of nine HC subjects and the FTD patient showed no evident $\mathrm{A} \beta$ deposition. Parametric group comparison showed that $\mathrm{A} \beta$ deposition was significantly greater in bilateral posterior cingulate gyri and precuneus in the AD group compared to the AZD-negative HC subjects (family-wise error corrected, $\mathrm{p}<0.05$ ). As for the MCI group, when parametric group comparison was performed using a more liberal statistical threshold (false discovery rate corrected, $\mathrm{p}<0.01$ ), $\mathrm{A} \beta$ deposition was significantly greater in the left middle occipital gyrus and bilateral precuneus than the AZD-negative HC subjects. Compared to [11 C]PIB PET images in our previous study, non-specific ligand binding to white matter was low in [11 C]AZD2184 PET. Furthermore, in patients with $\mathrm{A} \beta$ deposition, ligand binding to frontal cortex tended to be lower in [11 C]AZD2184 PET than those in [11 C]PIB PET. Conclusions: The present study demonstrated that $[11 \mathrm{C}] \mathrm{AZD} 2184 \mathrm{PET}$ is helpful to detect $\mathrm{A} \beta$ deposition in vivo. Patterns of distribution would be different between [11 C]AZD2184 and [11 C]PIB PET, suggesting that [11 C]AZD2184 and [11 C]PIB may detect different types of $\mathrm{A} \beta$ plaques.

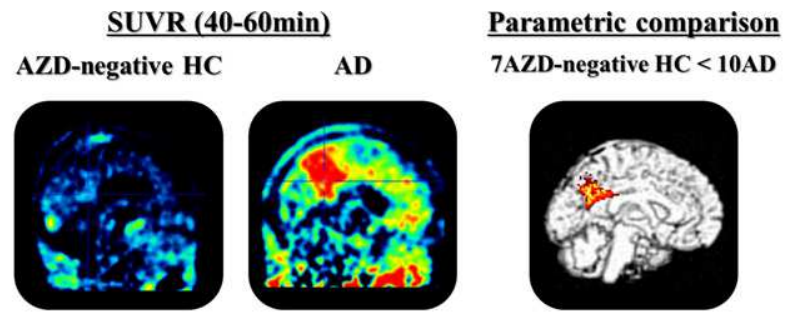

SUVR: standardized uptake value ratios to cerebellum as a reference $\mathrm{HC}$ : healthy control, AD: Alzheimer's disease

Family-wise error (FWE)-corrected $\mathrm{p}<0.05, \mathrm{k}>50$ voxels

Figure 1. Representative SUVR and parametric comparison.

\section{IC-P-026 DEFINING AN OPTIMAL APPROACH FOR EVALUATING REGIONAL METABOLISM WITH 18F-FDG-PET IMAGING: ANATOMICAL VERSUS PROBABILISTIC VOLUMES OF INTEREST}

Jean-Paul Soucy ${ }^{1}$, Ricardo Soder ${ }^{2}$, Pedro Rosa-Neto ${ }^{1}$, Serge Gauthier ${ }^{3}$, ${ }^{1}$ McGill University, Montreal, Quebec, Canada; ${ }^{2}$ McGill, Montreal, Quebec, Canada; ${ }^{3}$ McGill Centre for Studies in Aging, Montreal, Quebec, Canada.

Background: [18 F]FDG-PET is a recognized tool to identify abnormal patterns of brain metabolism (CMRglc) accompanying neurodegenerative diseases. Although pathological changes in $\mathrm{AD}$ affect first the mesial temporal lobes, the presence of abnormal CMRglc at that level at different disease stages remains controversial, possibly because of technical issues. We analyzed studies from subjects with normal cognition (NCo), with $\mathrm{MCI}$ and with $\mathrm{AD}$, using: 1) anatomically or 2) probabilistically defined volumes of interest (VOIs), to determine how to better identify CMRglc abnormalities. Methods: Subjects were categorized following standard criteria. FDG studies were spatially normalized to a PET template and intensity normalized to the pons. Four anatomically defined VOIs obtained from the template (cingulate gyrus $=\mathrm{CG}$, precuneus $=\mathrm{pCu}$, infraparietal cortex $=\mathrm{IPC}$, hippocampus $=\mathrm{HIP}$ ) were analyzed. Three probabilistic VOIs (posterior cingulate $=$ PCC, IPC, HIP) were created from t-statistical FDG-PET comparisons between $\mathrm{AD}$ and $\mathrm{NCI}$ cases, using voxels with a t value of at least 